\title{
Modelling of impurity transport and plasma-wall interaction in fusion devices with the ERO code: basics of the code and exam- ples of application
}

\author{
A. Kirschner ${ }^{1, *}$, D. Tskhakaya ${ }^{2,3}$, G. Kawamura ${ }^{4}$, D. Borodin ${ }^{1}$, S. Brezinsek ${ }^{1}$, R. Ding ${ }^{5}$, Ch. \\ Linsmeier $^{1}$, and J. Romazanov ${ }^{1}$ \\ ${ }^{1}$ Forschungszentrum Jülich GmbH, Institut für Energie- und Klimaforschung - Plasmaphysik, 52425 Jülich, \\ Germany \\ ${ }^{2}$ Institute of Applied Physics, TU-Wien, A-1040 Vienna, Austria \\ ${ }^{3}$ Institute of Theoretical Physics, University of Innsbruck, A-6020 Innsbruck, Austria \\ ${ }^{4}$ National Institute for Fusion Science, Toki 509-5292, Gifu, Japan \\ ${ }^{5}$ Institute of Plasma Physics, Chinese Academy of Sciences, P.O. Box 1126, Hefei, Anhui 230031, P.R.China
}

Received XXXX, revised XXXX, accepted XXXX

Published online XXXX

Key words fusion research, ERO, PIC, prompt deposition, erosion, plasma-wall interaction.

\begin{abstract}
The 3D ERO code, which simulates plasma-wall interaction and impurity transport in magnetically confined fusion-relevant devices is described. As application, prompt deposition of eroded tungsten has been simulated at surfaces with shallow magnetic field of $3 \mathrm{~T}$. Dedicated PIC simulations have been performed to calculate the characteristics of the sheath in front of plasma-exposed surfaces to use as input for these ERO simulations. Prompt deposition of tungsten reaches $100 \%$ at the highest electron temperature and density. In comparison to more simplified assumptions for the sheath the amount of prompt deposition is in general smaller if the PICcalculated sheath is used. Due to friction with the background plasma the impact energy of deposited tungsten can be significantly larger than the energy gained in the sheath potential.
\end{abstract}

Copyright line will be provided by the publisher

\section{Introduction}

The erosion of wall material in fusion devices is a crucial issue due to reduction of life time of wall components and also due to long-term tritium retention by means of tritium co-deposition together with the eroded material. At locations of highest power and particle loadings high-Z materials, in particular tungsten, are foreseen as plasma-facing material. Tungsten has a high melting point and exhibits comparably low physical sputtering. Therefore, the divertor of ITER will consist of tungsten based plasma-facing materials. An additional advantage of high- $\mathrm{Z}$ materials is the expected large amount of prompt deposition, i.e. deposition during the first gyration after being eroded. Due to their large number of electrons high- $\mathrm{Z}$ materials have rather large ionisation probabilities and due to their large mass also large gyration radii which increase the probability of prompt deposition. Within the present work the 3D impurity transport and plasma-wall interaction code ERO is used in combination with Particle in Cell (PIC) simulations to address the issue of prompt deposition of tungsten.

The second section gives a brief description of the ERO code. Particular attention is turned to the electrical sheath in front of plasma-exposed surfaces. The normally used simplified assumptions for the sheath will be compared with PIC simulations. ERO simulations of prompt deposition of sputtered tungsten are presented in the third section. A parameter study, also covering conditions at the ITER divertor, shows the dependence of prompt deposition on the electron temperature and density. Moreover, the mean energy and charge of deposited tungsten particles is discussed. Results for the sheath characteristics from PIC are used as input for ERO to analyse the prompt deposition of tungsten in comparison to the simplified assumptions.

\footnotetext{
* Corresponding author E-mail: a.kirschner@fz-juelich.de, Phone: +492461 61 4277, Fax: +49 2461612660
} 


\section{The ERO code}

ERO is a three-dimensional Monte-Carlo code to simulate plasma-wall interaction and impurity transport in fusion devices with magnetic confinement. Originally the code was developed in the beginning of the 1990s [1] but continuous development and application to a large number of experiments has been done. Typically, the simulations are focused on localized wall components with sizes in the range of $\mathrm{cm}$ to $\mathrm{m}$ like divertor target plates, limiter tiles or samples exposed to linear machines. Besides benchmarking simulations with existing experiments, see e.g. [2, 3, 4] also predictive modelling of the life time of wall components in ITER have been performed, see e.g. [5,6]. Currently the source code is subject of a major rewriting aiming at an improved performance. Together with a generalised definition of surfaces the revised ERO version should be capable of simulating much larger volumes than nowadays, as far as possible even whole machine dimensions of fusion experiments like divertor and main chamber of ITER. The modelling of the global migration of impurities thus will be an additional important application. In the following only the basic features of ERO will be described; more details can be found in $[7,8]$.

\subsection{Basic description of the ERO code}

Within a user-defined simulation volume around a wall component the background hydrogen plasma has to be provided as input. This in particular includes the electron temperature and density, the ion temperature and the magnetic field (strength and orientation). The electric field and the plasma flow velocity are typically calculated within the code depending on the plasma conditions. Alternatively, these two properties can be provided as input, e.g. originating from plasma simulations - more details will be given in section 2.2. The local transport of impurities generated at such surfaces is then treated in detail considering cross field diffusion, Lorentz force in combined electric and magnetic fields, friction with background ions due to Coulomb forces and thermal forces. Also collisions between impurity particles and background neutrals can be considered, which is important for conditions with large neutral pressure like for instance in linear devices. While moving through the background plasma the impurity particles can be ionised, recombine or, in case of molecules, be dissociated. Necessary rate coefficients for these processes are taken from literature. The transport simulation of an impurity particle ends if the particle leaves the simulation volume or is deposited on the surface.

Impurity test particles for which ERO simulates the transport through the plasma can be generated by various processes: physical sputtering, chemical erosion and background impurities reflected at the surface. The impurity content in the plasma has to be defined by input parameters. From the given electron temperature $T_{e}$ and density $n_{e}$ at the sheath entrance the incident flux $\Gamma$ of plasma ions is given by $\Gamma=n_{e} \cdot c_{S}\left(n_{e}, T_{e}\right) \cdot \sin (\alpha)$ with $c_{S}$ the sound speed of the plasma ions and $\alpha$ the angle between the magnetic field and the surface. A cross field flux can be added, which in particular becomes dominant for shallow angles $\alpha$. Typically the surface is divided into surface cells and test particles start from each surface cell. The flux of eroded impurities starting from each surface cell is given by $\Gamma \cdot Y \cdot c_{\text {solid }}$, where $Y$ is the yield for the specific erosion process and $c_{\text {solid }}$ the concentration of the impurity in the surface cell under consideration. The mixing of different species inside the surface and thus the concentrations of impurities $c_{\text {solid }}$ in the material is changing dynamically with plasma exposure. In ERO this is calculated by means of a simple homogeneous mixing model or alternatively a coupling with SDTrimSP is available. Details of both methods can be found in [9]. The number of different impurity species to be considered is in principle unlimited. The flux of reflected impurities from background impurity particles hitting the surface is given by $\Gamma \cdot R \cdot c_{\text {plasma }}$, with $R$ the reflection coefficient of the impurity on the surface and $c_{\text {plasma }}$ the impurity content in the plasma.

As the real number of impurity particles starting from each surface cell is much too large, only a certain number of test particles is simulated instead. The number of test particles is given by an input parameter and influences the computational time nearly linearly. In most cases it is assumed that the impurity fluxes from the surfaces are small enough not to influence the plasma parameters but fulfilling the test particle approximation. However, in specific cases like external injection of particles, the possible effects of local cooling and increase of plasma density has been considered, see [10]. Test particles returning to the surface can be reflected, generate new impurities by sputtering or can be deposited. An ERO simulation can be divided into time steps to take into account the dynamic changes of material mixing in the surface. 
For the various plasma-wall interaction and atomic processes diverse data bases for yields and rate coefficients are used. Sputtering and erosion yields are based on SDTrimSP and Molecular Dynamics simulations, if possible in combination with experimental data. Ionisation, recombination and photon emission rates for atoms are typically taken from ADAS [11]. Rate coefficients for molecules (e.g. hydrocarbons or BeD) come from separate calculations together with measurements.

\subsection{Description of the sheath}

In the default version of ERO details of the sheath are not resolved. Therefore values of the electron and ion density and temperature or the plasma flow velocity are given at the sheath entrance, which is then also the location of the surface. As the dimension of the sheath is very small - typically in the order of few Debye lengths or gyration radii of the plasma ions - this simplification is uncritical for many applications. However, this could be different e.g. when studying prompt deposition of tungsten within a strongly magnetised plasma as the ionisation length of eroded tungsten atoms can be smaller than the dimension of the sheath - this will be analysed in the subsequent section 3 .

For the simplified sheath described in a two point model, the electron density decreases by a factor of 2 when moving from the stagnation point to the sheath entrance (surface) and the plasma flow velocity increases from zero to sound speed. The electrical sheath potential $U_{S}$ scales exponentially with the Debye length $\lambda_{D}$ if the magnetic field is perpendicular to the surface. For shallow magnetic field lines the potential decays exponentially with the gyration radius $r_{\text {gyro }}$.

$$
U_{S}=U_{0} \cdot f(\alpha) \cdot \exp \left(-\frac{z}{2 \lambda_{D}}\right)+U_{0} \cdot(1-f(\alpha)) \cdot \exp \left(-\frac{z}{r_{\text {gyro }}}\right)
$$

$U_{0}$ is the sheath potential drop, $f(\alpha)$ is a function with $f\left(\alpha=0^{\circ}\right) \rightarrow 0$ and $f\left(\alpha=90^{\circ}\right)=1$ and $z$ is the distance from the surface. In a simplified evaluation with $T_{e}=T_{i}$ and neglecting secondary electron emission, $U_{0} \approx 3 T_{e}$, here $U_{0}$ is given in [V] for $T_{e}$ in [eV]. For the total potential, a pre-sheath drop of $\approx 0.7 T_{e}$ has to be added to satisfy the Bohm-criterion.

PIC simulations can provide more detailed characteristics of the sheath. In the present work the BIT1 code [12] has been used to study the sheath with magnetic field $B=3 \mathrm{~T}$ and $\alpha=2^{\circ}$. Exemplary results are presented here for an electron density of $6 \mathrm{E} 19 \mathrm{~m}^{-3}$ and temperature of $20 \mathrm{eV}$ at the sheath entrance. Figure 1 shows the resulting electron density in front of the surface in comparison with the simple sheath, for the latter one the stagnation point is assumed to be $10 \mathrm{~m}$ away from the surface. The PIC simulations show a significant decrease of the density when approaching the surface from the sheath entrance (indicated as magenta line in figure 1 at about $8 \mathrm{~mm}$ away from the surface) whereas the decrease for the simple case is marginal due to the large distance of $10 \mathrm{~m}$ to the stagnation point.

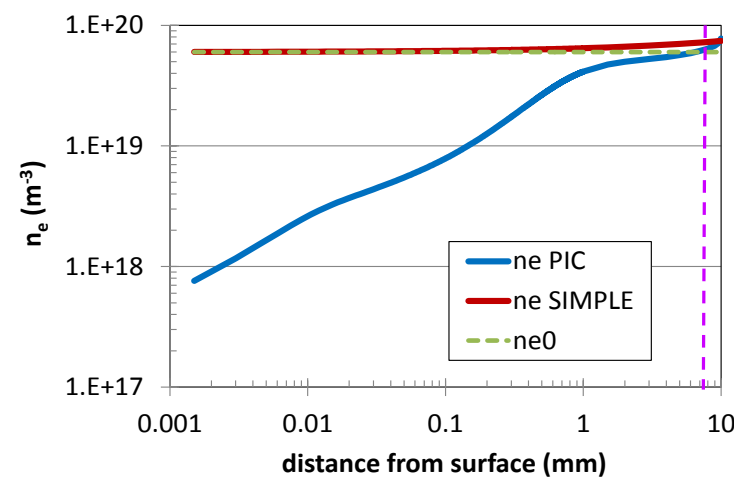

Fig. 1 Electron density within the sheath: PIC vs. SIMPLE assumption. The magenta line indicates the location of sheath entrance. ne $0=6 \mathrm{E} 19 \mathrm{~m}^{-3}$ is the nominal value of the density at the sheath entrance.

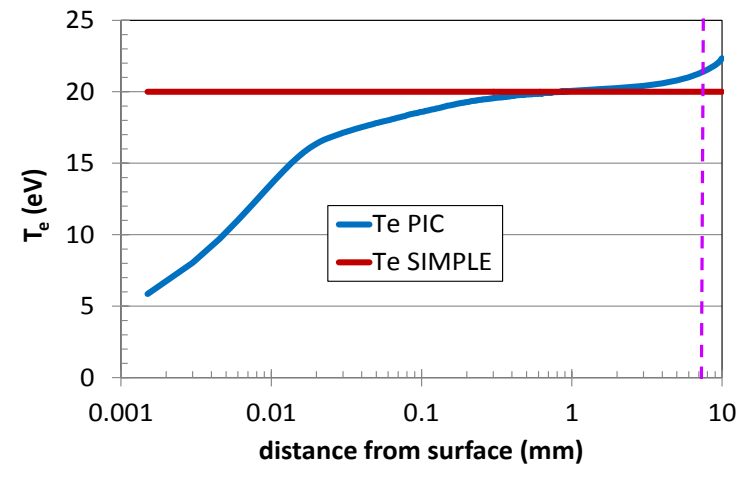

Fig. 2 Electron temperature within the sheath: PIC vs. SIMPLE assumption. The magenta line indicates the location of sheath entrance. The nominal temperature at the sheath entrance is $20 \mathrm{eV}$. 
In any case, the density at the surface resulting from PIC is much smaller than in the simple case where we have per definition $6 \mathrm{E} 19 \mathrm{~m}^{-3}$ at the surface. Figure 2 presents the electron temperature $T_{e}$ depending on the distance from the surface. Whereas $T_{e}$ from PIC decreases from $20 \mathrm{eV}$ at the sheath entrance to about $3 \mathrm{eV}$ at the surface, it is constant for the simple sheath. From these results one can expect that the sheath details can become important for the estimation of tungsten deposition if the ionisation length of sputtered tungsten is clearly smaller than the extension of the sheath such that variations of $n_{e}$ and $T_{e}$ become noticeable.

\section{Modelling of tungsten deposition}

For the ERO simulations a roof-like surface geometry with an inclination angle of $2^{\circ}$ is used, see figure 3 . Tungsten atoms are injected at the middle point of the surface with a Thompson energy distribution around $8 \mathrm{eV}$ (describing the energy distribution of sputtered tungsten) and a cosine angular distribution. The electron density and temperature are given as input at the stagnation point, which is $10 \mathrm{~m}$ away from the surface. As no final ADAS data are available yet, ionisation of tungsten atoms $\mathrm{W}^{0}$ and singly charged ions $\mathrm{W}^{+}$is calculated with rate coefficients from [13], for higher ionised $W$ atoms the Lotz formula is used [14]. Given that for the simple sheath assumption the electron and ion temperature inside the sheath are constant along the magnetic field lines, the thermal force is calculated using formulae for the temperature distribution and resulting thermal force from literature [15]. Self-sputtering of deposited tungsten particles is not considered.

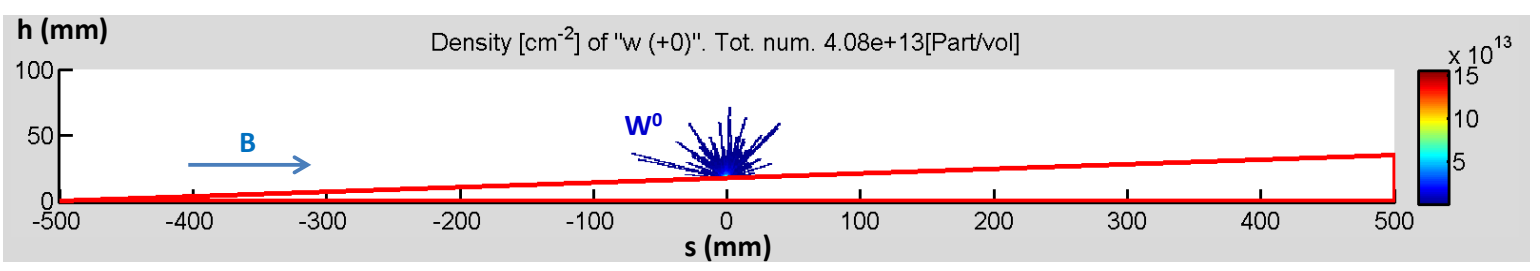

Fig. 3 Set-up for the ERO simulations with an example of simulated tungsten injection (2D spatial distribution of injected atoms above the surface, integrated in y-direction); $n_{e}=1 \mathrm{E} 19 \mathrm{~m}^{-3}$ at the stagnation point and $T_{e}=5 \mathrm{eV}$, B field angle: $2^{\circ}$.

\subsection{Dependence of prompt deposition on electron temperature and density for simple sheath}

Using the simplified assumption for the sheath ERO, simulations have been performed for electron temperatures between $1 \mathrm{eV}$ and $20 \mathrm{eV}$ and electron densities between $1 \mathrm{E} 18 \mathrm{~m}^{-3}$ and $1 \mathrm{E} 21 \mathrm{~m}^{-3}$ at the stagnation point. The calculated amounts of tungsten prompt deposition relative to the amount of injected tungsten atoms are summarised in figure 4. As expected, prompt deposition increases with increasing electron temperature and density. For the highest density and temperature studied, $100 \%$ of injected tungsten is deposited promptly. For 1 $\mathrm{eV}$ and electron densities up to $1 \mathrm{E} 20 \mathrm{~m}^{-3}$ no prompt deposition takes place and even at the highest density and an electron temperature of $1 \mathrm{eV}$ only $2 \%$ prompt deposition occurs.

Figures 5 and 6 present the mean energy and charge of promptly deposited tungsten. For the highest density and small temperatures the mean energy $E_{\text {mean }}$ is significantly larger than expected from the sheath potential, $E_{\text {mean }} \approx 3 Q_{\text {mean }} T_{e}$ with $Q_{\text {mean }}$ the mean charge. This is the result from very effective friction of $\mathrm{W}$ ions with the plasma ions at the high density. In contrast, at high temperature and high density the mean energy is smaller than expected from the sheath potential. In these cases the ionisation length of tungsten is very small such that the sheath potential is not completely passed. The mean charge of promptly deposited tungsten is mainly increasing with density reaching a maximum value of nearly 2 at the maximal density and temperature studied.

\subsection{ERO simulations of prompt deposition with sheath characteristics from PIC}

The resulting distribution of density, temperature, flow velocity, electric field and thermal force within the sheath from PIC has been used as input for ERO for the example cases of $n_{e}=6 \mathrm{E} 19 \mathrm{~m}^{-3}$ and $T_{e}=20 \mathrm{eV}$ or $5 \mathrm{eV}$ at the sheath entrance. The main results are summarised in table 1. With ERO-PIC the amount of prompt tungsten deposition decreases from $99 \%$ to $95 \%$ for $20 \mathrm{eV}$ and from $73 \%$ to $65 \%$ for $5 \mathrm{eV}$. The mean charges of promptly deposited tungsten are similar $(1.3-1.5$ for $20 \mathrm{eV}$ and $1.0-1.1$ for $5 \mathrm{eV})$. 


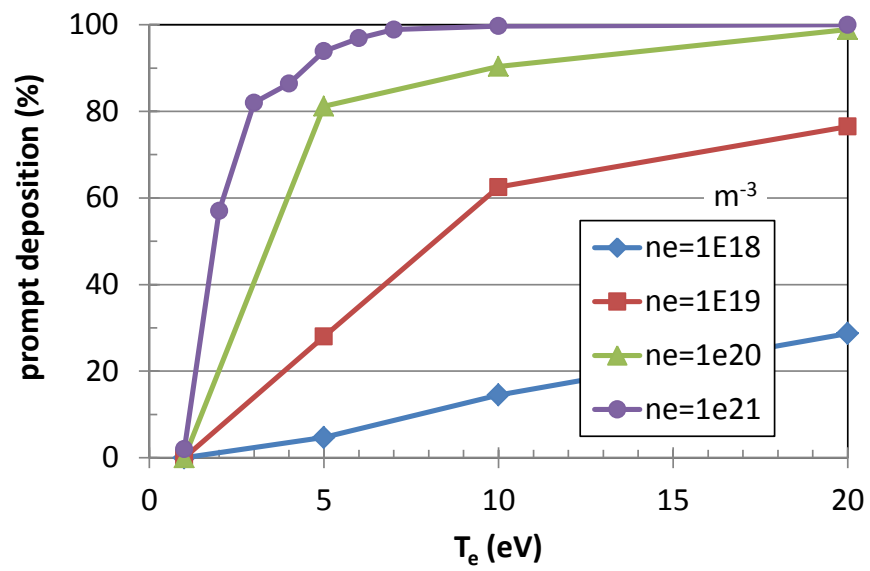

Fig. 4 Simulated amount of prompt deposition for tungsten in dependence on the electron temperature for various electron densities employing the simple sheath assumptions. Self-sputtering of tungsten is neglected

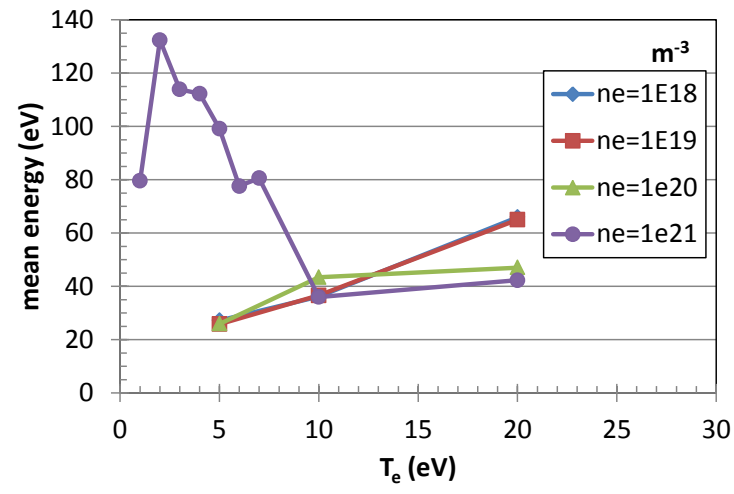

Fig. 5 Simulated mean energy of promptly deposited tungsten (simple sheath assumption).

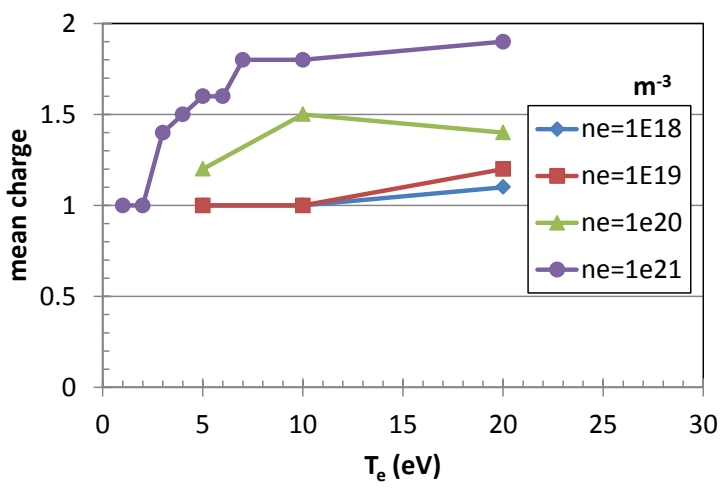

Fig. 6 Simulated mean charge of promptly deposited tungsten (simple sheath assumption).

However, the mean impact energy of promptly deposited tungsten from ERO-PIC for the $20 \mathrm{eV}$ case is with $E_{\text {mean }}=89 \mathrm{eV}$ significantly larger than for ERO with simple sheath $\left(E_{\text {mean }}=24 \mathrm{eV}\right)$. In contrast, for the $5 \mathrm{eV}$ case similar energies of about $E_{\text {mean }}=15 \mathrm{eV}$ are calculated. The tungsten ionisation for the $20 \mathrm{eV}$ case with the simple sheath occurs very near to the surface such that returning particles do not see the full sheath potential. In contrast, as the density and temperature decrease approaching the surface within the sheath in the ERO-PIC case (see figures 1 and 2), ionisation takes place farer away from the surface such that the whole sheath potential is traversed by deposited tungsten ions finally leading to larger energies. For the $5 \mathrm{eV}$ case ionisation takes place outside the sheath potential drop both for ERO-PIC and ERO with simple sheath such that the mean impact energy of deposited tungsten corresponds to $\approx 3 Q_{\text {mean }} T_{e}$.

Table 1 Simulated amount of tungsten prompt deposition, mean energy and mean charge of promptly deposited tungsten particles: ERO with simple sheath vs. ERO-PIC.

\begin{tabular}{lllllll}
\hline & \multicolumn{3}{c}{ ERO with simple sheath } & \multicolumn{3}{c}{ ERO-PIC } \\
& $\begin{array}{l}\text { Prompt } \\
\text { Seposition }\end{array}$ & $\begin{array}{l}\text { Mean } \\
\text { energy }\end{array}$ & $\begin{array}{l}\text { Mean } \\
\text { charge }\end{array}$ & $\begin{array}{l}\text { Prompt } \\
\text { deposition }\end{array}$ & $\begin{array}{l}\text { Mean } \\
\text { energy }\end{array}$ & $\begin{array}{l}\text { Mean } \\
\text { charge }\end{array}$ \\
\hline $6 \mathrm{E} 19 \mathrm{~m}^{-3}, 20 \mathrm{eV}$ & $99 \%$ & $24 \mathrm{eV}$ & 1.3 & $95 \%$ & $89 \mathrm{eV}$ & 1.5 \\
$6 \mathrm{E} 19 \mathrm{~m}^{-3}, 5 \mathrm{eV}$ & $73 \%$ & $15 \mathrm{eV}$ & 1.1 & $65 \%$ & $16 \mathrm{eV}$ & 1.0 \\
\hline
\end{tabular}




\section{Conclusions and summary}

It has been seen that entrainment, thus acceleration of ions by means of friction with the background plasma, at large plasma densities can lead to energies of deposited particles much larger than expected from the sheath potential drop - this has to be considered when sputtering by deposited particles is studied. In particular, the possible effect of self-sputtering avalanche has to be analysed in detail for promptly and also for non-promptly deposited particles. The impact of entrainment is more distinctive for non-prompt deposition as these particles travel over larger distances before hitting the surface. Sheath-resolved characteristics, in the present work calculated by PIC simulations and then used as input in ERO, can become important for very small ionisation lengths of eroded particles and potentially reduce the amount of prompt deposition. If ionisation takes place mainly within the sheath, the amount of ERO-PIC simulated prompt deposition of tungsten for the cases studied so far is similar to values estimated in [16] by means of an analytic fit formula which has been deduced for the condition when ionisation takes place inside the sheath. In the future more studies will be done with ERO-PIC in comparison with results from [16] and also recent work published in [17] carried out by Monte-Carlo simulations.

Acknowledgements This work has been carried out within the framework of the EUROfusion Consortium and has received funding from the Euratom research and training programme 2014-2018 under grant agreement No 633053. The views and opinions expressed herein do not necessarily reflect those of the European Commission. This work has been done under WP PFC. It has been supported by the Sino-German Center for Research Promotion under Contract No. GZ769. The second author acknowledges support by the project FWF P26544-N27.

\section{References}

[1] D. Naujoks, R. Behrisch, J.P. Coad and L. de Kock, Nucl. Fusion 33 (1993) 582.

[2] A. Kirschner, P. Wienhold, V. Philipps et al., J. Nucl. Mat. 328 (2004) 62.

[3] D. Borodin, R. Doerner, D. Nishijima et al., J. Nucl. Mat. 415 (2011) S219.

[4] A. Kirschner, D. Matveev, D. Borodin et al., J. Nucl. Mat. 463 (2015) 116.

[5] D. Borodin, A. Kirschner, S. Carpentier-Chouchana et al., Phys. Scr. T145 (2011) 014008.

[6] A. Kirschner, D. Borodin, S. Droste et al., J. Nucl. Mat. 363-365 (2007) 91.

[7] U. Kögler, J. Winter, ERO-TEXTOR: 3D Monte-Carlo code for local impurity modeling in the scrape-off layer of TEXTOR, Jülich Report, Jül-3661 (1997).

[8] A. Kirschner, V. Philipps, J. Winter, U. Kögler, Nucl. Fusion 40 (2000) 989.

[9] S. Droste, A. Kirschner, D. Borodin, A. Kirschner et al., Plasma Phys. Control. Fus. 50 (2008) 015006.

[10] R. Ding, A. Kirschner, M.Z. Tokar, M. Koltunov et al., J. Nucl. Mat. 415 (2011) S270.

[11] H.P. Summers, The ADAS User Manual version 2.6 (2004), http://adas.phys.strath.ac.ukADAS.

[12] D. Tskhakaya, M. Groth, J. Nucl. Mat. 438 (2013) S522.

[13] L.A.Vainshtein, I. Beigman, Ph. Mertens et al., J. Phys. B: At. Mol. Opt. Phys. 44 (2011) 125201.

[14] M. Higgins et al., Atomic and molecular data for fusion, Part III: recommended cross sections and rates for electron ionisation of light atoms and ions: copper to uranium, Tech. Rep. CLM-R294, Culham Laboratory, Abingdon, Oxfordshire, UK (1989).

[15] P.C. Stangeby, J.D. Elder, Nucl. Fusion 35 (1995) 1391

[16] D. Tskhakaya, M. Groth, JET EFDA contributors, J. Nucl. Mat. 463 (2015) 624.

[17] A.V. Chankin, D.P. Coster, R. Dux, Plasma. Phys. Control. Fusion 56 (2014) 025003. 